\title{
Return to Rugby After Brain Concussion: A Prospective Study in 35 High Level Rugby Players
}

\author{
Jean Francois Chermann ${ }^{1, *}$; Shahnaz Klouche ${ }^{2,3}$; Alexis Savigny ${ }^{4}$; Nicolas Lefevre ${ }^{2,3}$; Serge \\ Herman $^{2,3}$; Yoann Bohu ${ }^{2,3,5}$ \\ ${ }^{1}$ Department of Neurology, Leopold Bellan Hospital, Paris, France \\ ${ }^{2}$ Department of Orthopedic Surgery, Musculoskeletal Institute of Nollet, Paris, France \\ ${ }^{3}$ Department of Orthopedic Surgery, Paris Sports Clinic, Paris, France \\ ${ }_{4}^{4}$ Medical Staff, Stade Francais Rugby, Paris, France \\ 5 Medical Staff, Racing-Metro 92, Le Plessis-Robinson, France \\ ${ }^{*}$ Corresponding author:Jean Francois Chermann, Department of Neurology, Leopold Bellan Hospital, Paris, France. Tel:+33-140486868, Fax: +33-143219813, e-mail:jfchermann@gmail.com
}

Received: October 20, 2013; Accepted: July 1, 2014

Background: Although guidelines based on expert opinions have been developed for the immediate management and return to play of athletes after a concussion, data are lacking on this issue.

Objectives: Evaluate a standardized management of brain concussion among rugby players to prevent the recurrence.

Patients and Methods: A prospective study was performed from September 2009 to June 2012. All rugby players who had a concussion when playing rugby were included. Patients were managed by a specialized hospital team with a specific protocol developed in collaboration with the medical staff of the rugby clubs included in the study. The series included 35 rugby players, with 23 professionals and 12 high-level players, 30 men and 5 women, mean age $23.1 \pm 5.5$ years old. The median number of previous concussions was 2 (0-30) episodes. According to the Cantu concussion severity classification, 3 athletes were grade 1, 12 were grade 2 and 20 were grade 3 . None of the injured athletes was lost to follow-up. The primary endpoint was the occurrence of a new concussion within 3 months after the first in patients who returned to rugby.

Results: Thirty-three patients returned to rugby after a mean $22.1 \pm 10$ days. The recurrence rate within 3 months was $2 / 33$ (6.1\%). The median delay before returning to rugby was 21 (7-45) days. Factors associated with a delayed return to play were young age, initial loss of consciousness, severity Cantu grade 3 and post-concussive syndrome of more than 5 days. Analysis of two failures showed that the initial injury was grade 3 and that both were professional athletes and had a history of concussion.

Conclusions: This prospective study validated the study protocol for the management of concussion in rugby players.

Keywords:Brain Concussion; Rugby; Recurrence; Trail Making Test; Post-concussion syndrome; Athletics Injuries

\section{Background}

Rugby is a team contact sport that is popular in many countries. In France, there were more than 390,000 licensed players at the French Rugby Union in 2010 including $4.5 \%$ women, with an increase in membership of $40 \%$ in the last five years. Called the "hidden epidemic" (1), brain concussion is a poorly understood entity, which is frequent but under-reported, serious and costly for society. This type of head trauma, which is considered to be slight, is secondary to a shock which results in a blowcounterblow of the brain against the skull, whatever the point of impact, but the biomechanical causes are more complex. It results in an immediate and transitory change in higher brain functions which may range from simple confusion to loss of consciousness (LOC) or knockout (KO) (2). Less than $10 \%$ of sports induced concussions result in LOC (3). Because of this, many traumas may not be diagnosed, and may be hidden by the player who does not wish to be taken out of play. All sports are implicated (4). In rugby, tackling is the most frequent mechanism of concussions $(5,6)$ while in soccer it frequently occurs in athletes when they are fighting in the air to head the ball (head to head) (7).

In the literature the incidence of concussions in rugby varies from 0.62-9.05 per 1,000 hours of play (8-10), with a higher risk in professional players. Women also seem to be at greater risk than their male colleagues (11) and take longer to recover (12). The incidence has increased significantly in the last twenty years (13) possibly because of better identification of concussions thanks to information campaigns to players and other participants in the field as well as an increase in the physical strength of athletes (14). 
Although the initial prognosis of brain concussion is reassuring, significant short and long term complications may occur (15-20). Several expert consensus conferences have been held on this subject $(21,22)$. Recently the "declaration of the Zurich consensus on brain concussion in sports " (2) provided updated guidelines for doctors on the initial diagnosis of concussion, immediate management of patients and management of the decision to return to sports.

\section{Objectives}

A standardized protocol based on the Zurich international guidelines was developed including a consult with a neurologist, so that patients could return to sports under optimal conditions to prevent recurrent concussion. The main aim of this study was to evaluate our protocol in a group of rugby players. The study hypothesis was that no recurrence of concussion would occur if patients are compliant with this protocol.

\section{Patients and Methods}

From September 2009 to June 2012 a prospective nonrandomized non-comparative study was performed in the neurology unit based on a specialized "sports concussion" hospital consultation. Concussed athletes were fully informed and the study was approved by the ethics committee as a non-interventional study.

\subsection{Inclusion and Exclusion Criteria}

All rugby players, both professional and amateur, who suffered a suspected brain concussion while playing rugby and confirmed by our consultation with one neurologist, were included. Among 14 professional and high level national rugby clubs, 3 clubs participated in the study. Concussion was suggested on the playing field by the club physician in the presence of confusion or memory disturbances (23) following a direct, violent shock such as a collision or tackling associated or without loss of consciousness. These symptoms were investigated using the 5 Maddocks (24) questions with one wrong answer justifying removal from the field. The exclusion criteria were patients who refuse to participate in the study and age under 18 years. No patient has refused to participate in the study.

\subsection{Management Protocol}

The protocol was developed in collaboration with the medical staff of the rugby clubs that participated in the study. In case of suspected concussion, the protocol was the following:

1) Immediate removal from the field, and total rest for the patient with concussion;

2) Computed tomography (CT) Scan or brain magnetic resonance imaging (MRI) to exclude any structural brain lesions (25);
3) A rapid initial consultation with the same neurologist no more than three days after the trauma for:

a) Clinical confirmation of the diagnosis and an estimation of symptoms in the patient on the SCAT2 scale (Sport Concussion Assessment Tool) (2);

b) Evaluation of the severity of the concussion according to the Cantu classification (23);

c) Evaluation of cognitive symptoms with the Trail Making Test (26) (TMT-part A and TMT-part B);

4) Return to exercising in stages managed by the club physician once post-concussive symptoms at rest have disappeared: first walking or biking then individual running, then training without contact;

5) In the absence of recurrent symptoms during exercising, a second specialized consultation confirming recovery based on the disappearance of cognitive disturbances on TMT tests. The neurologist could then approve a return to competitive sports.

Table 1. Evidence-Based Cantu Grading System for Concussion (23)

\begin{tabular}{|c|c|}
\hline Grade & Evidence \\
\hline Grade 1 (mild) & $\begin{array}{l}\text { No loss of consciousness; posttraumatic } \\
\text { amnesia a or postconcussion signs or } \\
\text { symptoms lasting less than } 30 \text { minutes }\end{array}$ \\
\hline Grade 2 (moderate) & $\begin{array}{c}\text { Loss of consciousness lasting less than } \\
1 \text { minute; posttraumatic amnesia }{ }^{\mathrm{a}} \text { or } \\
\text { postconcussion signs or symptoms } \\
\text { lasting longer than } 30 \text { minutes but less } \\
\text { than } 24 \text { hours }\end{array}$ \\
\hline Grade 3 (severe) & $\begin{array}{c}\text { Loss of consciousness lasting more than } \\
1 \text { minute or posttraumatic amnesia a } \\
\text { lasting longer than } 24 \text { hours; postcon- } \\
\text { cussion signs or symptoms lasting } \\
\text { longer than } 7 \text { days }\end{array}$ \\
\hline
\end{tabular}

${ }^{a}$ Retrograde and anterograde.

\subsection{Evaluation Criteria}

The main judgment criterion was another concussion within 3 months after returning to rugby. Secondary criteria were the delay before returning to rugby and the risk factors of a delayed return.

\subsection{Statistical Method}

Normal distribution was tested by the Shapiro-Wilk test. If distribution was normal, parametric tests were used: the Student t test for independent or paired groups for quantitative variables and the Chi2 or the MacNemar test for qualitative variables. If distributions were not normal, non-parametric tests were used: the Mann-Whitney test or the Wilcoxon test for continuous variables and the Fisher exact or the MacNemar exact test for binary variables. A Kruskal-Wallis test was performed to compare 
the delay between Cantu severity groups. The risk factors for a delayed return to rugby were investigated by univariate then multivariate logistic regression. A P value of less than 0.05 was considered to be statistically significant.

\section{Results}

The series included 35 patients, 30 men and 5 women, mean age $23.1 \pm 5.5$ years old. All patients were competitive athletes, 29 high level and 23 professionals; 19/35 (54.3\%) played "Back player" positions and 16/35 (45.7\%) patients were "Forward players". This difference was not significant. In 13/35 (37.1\%) patients, this concussion was the first while $7 / 35$ (20\%) had a history of 1 or 2 other concussions and 15/35 (42.9\%) patients had had 3 concussions or more. The median number of previous concussions was 2 episodes (range, 0-30). The diagnosis was usually suggested in the field by anterograde amnesia (Table 2). Brain imaging was strictly normal in all patients. The most frequent symptoms of post-concussive syndrome were headache and fatigue (Table 3 ).

Overall, this syndrome lasted a median of 6 days (range, 0.5-270), 15 days (range, 10-210) in women and 4 (range, $0.5-270$ ) in men $P=0.04$. According to the Cantu severity classification, 3 patients were grade 1, 12 were grade 2 and 20 were grade 3 . The initial mean TMTA was $30.8 \pm$ 25.4 (range, 13-126.6) seconds and $19.7 \pm 7.2$ (range, 12-29) seconds before returning to sports, which was not significant. The mean initial TMTB was $50.3 \pm 22.7$ (range, 20102 ) seconds and $44.4 \pm 17.5$ (range, 22-87) seconds before returning to sports $\mathrm{P}=0.01$. All patients consulted the neurologist before returning to sports and were followed up at least 3 months after returning to rugby. The rate of recurrent brain concussion within 3 months was 2/33 (6.1\%) in the 33/35 (94.3\%) patients who returned to rugby. The median delay before returning to sports was 21 days (range, 7-45) and varied according to Cantu severity classification (Table 4).

This delay was longer in women (30, range 18-35) than in men (21, range 7-45) but this was not statistically significant $(P=0.19)$. Thirteen of thirty three patients $(39.4 \%)$ returned to rugby in less than 21 days including 1 against the advice of the doctor, and 20/33 patients (60.6\%) returned to rugby in 21 days or more after the concussion. With univariate analysis the risk factors of a delayed return to rugby (more than 21 days) were young age (20.7 \pm 5.3 vs. $26.1 \pm 4.9, P=0.01)$, initial loss of consciousness ( $P$ $=0.03)$, Cantu grade $3(P=0.02)$ concussion and a postconcussive syndrome of more than 5 days $(P=0.03)$. With multivariate analysis only a Cantu grade 3 concussion was significant $(P=0.01)$. Analysis of the two failures showed that the initial concussion was grade 3 in these professional athletes who both had a history of concussion (Table 5).
Table 2. Clinical Signs of Brain Concussion at the Initial Examination

\begin{tabular}{lc}
\hline Variable & Value \\
\hline Anterograde Amnesia $^{\mathrm{a}}$ & $29 / 35(82.9 \%)$ \\
Median Duration, min $^{\mathrm{a}}$ & $60(5-600)$ \\
Loss of Consciousness $^{\mathrm{a}}$ & $17 / 35(48.6 \%)$ \\
Retrograde Amnesia $^{\mathrm{a}}$ & $8 / 35(22.9 \%)$ \\
Median Duration, min & $37.5(5-2000)$ \\
\hline
\end{tabular}

a Data are presented as No. (\%).

\begin{tabular}{|c|c|}
\hline Symptoms & Frequency \\
\hline Headache & $29 / 35(82.9 \%)$ \\
\hline Fatigue & $20 / 35(57.1 \%)$ \\
\hline Sensitivity to light & $10 / 35(28.6 \%)$ \\
\hline Balance disturbances & $10 / 35(28.6 \%)$ \\
\hline Memory disturbances & $10 / 35(28.6 \%)$ \\
\hline Sensitivity to noise & $8 / 35(22.9 \%)$ \\
\hline Hypersomnia & $8 / 35(22.9 \%)$ \\
\hline Mood disturbances ${ }^{b}$ & $6 / 35(17.1 \%)$ \\
\hline Insomnia & $5 / 35(14.3 \%)$ \\
\hline Visual disturbances & $5 / 35(14.3 \%)$ \\
\hline Cervicalgia & $4 / 35(11.4 \%)$ \\
\hline Malaise & $2 / 35(5.7 \%)$ \\
\hline Neurotic disorders ${ }^{\mathrm{C}}$ & $1 / 35(2.9 \%)$ \\
\hline
\end{tabular}

Table 4. Delay Before Returning to Sports According to Cantu Severity Classification

\begin{tabular}{lcccc}
\hline & $\begin{array}{c}\text { Cantu 1 } \\
(\mathbf{n}=\mathbf{3})\end{array}$ & $\begin{array}{c}\text { Cantu 2 } \\
(\mathbf{n}=12)\end{array}$ & $\begin{array}{c}\text { Cantu 3 } \\
(\mathbf{n}=\mathbf{2 0})\end{array}$ & P Value \\
\hline Delay, d & & & & 0.004 \\
7 & 2 & 0 & 1 & \\
$8-14$ & 1 & 4 & 0 & \\
$15-20$ & 0 & 2 & 3 & \\
$>21$ & 0 & 6 & 14 & \\
\hline Returned to sports & 3 & 12 & 18 & \\
\hline
\end{tabular}

Moreover, one of these patients who had already had 30 concussions returned to rugby after 7 days against the advice of the physician. The neurologist advised two 
Chermann JF et al.

\begin{tabular}{lcc}
\hline Table 5. Characteristics of Patients With Recurrent Concussion & Patient 1 & Patient 2 \\
\hline Variable & Male & Male \\
\hline Gender & 28 & 28 \\
$\begin{array}{l}\text { Age, } \mathbf{y} \\
\text { Position }\end{array}$ & Back (fly half) & Forward (3rd) \\
Level of play & High level professional & High level professional \\
Number of previous concussions & 30 & 2 \\
Signs at the initial examination & Loss of consciousness, Anterograde amnesia & Loss of consciousness \\
Cantu severity grade & 3 & 3 \\
Post concussive syndrome & Headache, Anterograde amnesia & Headache, Asthenia, Balance disturbanc- \\
& & es, Memory disturbances \\
Duration of post concussive syndrome, $\mathbf{d}$ & 2 & 7 \\
Delay before returning to rugby, & 7 & 28 \\
\hline
\end{tabular}

other patients to discontinue rugby permanently, 1 man and 1 woman, respectively aged 24 and 27 years old who were high level professionals; one was a Forward player and the other a Back player (center). Both had a history of multiple concussions ( 3 and 10). The concussion that was managed in this study was grade 3 . This decision was based on persistent headaches after the slightest contact in one, and dizziness and balance disturbances in the other.

\section{Discussion}

In this study only two patients presented with another concussion within 3 months after they returned to rugby following the initial injury, including one player who did not respect the stopping delay and began playing rugby again against the advice of the physician. This protocol also identified two at risk patients who the neurologist advised to stop playing rugby altogether. It is important to note that these two patients had not stopped playing rugby after their previous concussions before our consultation. They had had early and multiple recurrent concussions, and developed an increased susceptibility to concussion.

Makdissi et al. (27) analyzed the conditions under which a group of Australian soccer players returned to sports after concussion. After 6-9 days off the field, 127/138 (92\%) patients with concussion began playing soccer again, without missing a match, and the remaining $8 \%$ missed one match. Cognitive tests showed a clear improvement before returning to sports. There were no recurrent concussions during the first match athletes played when they returned to soccer. In our series with rugby players, more than $94 \%$ of the patients returned to rugby. Moreover our judgment criteria included a longer, 3-month follow up period, which seems better adapted to determine recurrent concussion. The known risk factors of recurrence are failure to wear a helmet and a history of concussion (9). The patients in our series who had recurrent concussion within three months after returning to sports also had a history of concussion. In the literature the risk factors for a delayed return to rugby were younger age, initial loss of consciousness, a headache that lasts more than 3 hours and retrograde amnesia $(28,29)$. Women have been reported to take longer to return to sports (6) but this was not found in all studies (30). Our study showed that the stage of severity and the duration of the post-concussive syndrome were also factors. Women took longer to return to play than men but this was not significant.

There are numerous scales to assess concussions and their severity (31), and we used SCAT2. Neuropsychological tests play an important role in the initial management of concussions but also in the recommendation to return to sports. A battery of computerized tests such as ImPACT (32) is recommended in North America to evaluate ice hockey and American football players. These tests measure response time in milliseconds and have thousands of random combinations. However tests on paper such as the Trail Making Test (26) are also appropriate: initiation is tested by part A of the TMT and flexibility, or the capacity to displace the focus of attention from one class of stimuli to another, by part B of the TMT. In our study a significant improvement in the TMTB was observed before returning to sports. However, Makdissi et al. (33) found a 2-3 day delay in normalization between electronic tests and paper tests, which they feel is a sign of greater sensitivity of computerized tests.

Several studies suggest that the decision recommendation to return to sports should be made by an independent neurologist $(34,35)$ who has no particular interest in approving a premature return to sports, preventing players or trainers from putting pressure on the club physician. However, most places do not have a neurologist to do consultations on all concussed athletes, especially for amateur clubs. A well-trained sports physician should be able to provide the same level of care according to the same rigorous protocol, only referring the more complex cases to the neurologist.

Although information campaigns in clubs focused on players and their families have helped improve understanding of this entity (36) and increased the number of 
declarations, in particular by trainers on the field (37), compliance to guidelines is still insufficient $(38,39)$. Until 2011, for the International Rugby Board (IRB), a player who was removed from the field for concussion had to stop playing rugby for 3 weeks. Early return to play was only possible if a specialist filled out a certificate. This had two consequences: first under-reporting of accidents to avoid being out of play for 3 weeks, and in case of a reported concussion, the necessity of finding medical elements to approve a return to play. Since 2011 other guidelines have been published in France, including in particular, pre-season clinical tests (40) while, the 3-week protection rule was shortened in 2011 by the IRB.

This study has several limitations. One of the main criteria for the decision to return to sports was the resolution of cognitive disturbances but the ideal would have been to compare with preseason cognitive tests. This will be possible from now on the professional rugby players. The rate of recurrent concussion was low, but must be confirmed in a larger study. Risk factors for recurrence could not be statistically determined, because there were very few failures.

Brain concussions are frequent during contact sports and often under-reported. On the other hand, the consequences are potentially serious and may present a health risk or compromise an athlete's career. This prospective study provides a unique description of management of this entity in a population of high level rugby players. Athletes with a concussion only returned to sports when clinical and paraclinical signs had normalized. Young age, initial grade of concussion and initial loss of consciousness significantly delay a return to sports rugby.

\section{References}

1. Marshall SW, Spencer RJ. Concussion in Rugby: The Hidden Epidemic. J Athl Train. 2001;36(3):334-8.

2. McCrory P, Meeuwisse W, Aubry M, Cantu B, Dvorak J, Echemendia RJ, et al. Consensus statement on concussion in sport-the 4th International Conference on Concussion in Sport held in Zurich, November 2012. Clin J Sport Med. 2013;23(2):89-117.

3. Meehan WP, 3rd, d'Hemecourt P, Comstock RD. High school concussions in the 2008-2009 academic year: mechanism, symptoms, and management. Am J Sports Med. 2010;38(12):2405-9.

4. Lincoln AE, Caswell SV, Almquist JL, Dunn RE, Norris JB, Hinton RY. Trends in concussion incidence in high school sports: a prospective 11-year study. Am J Sports Med. 2011;39(5):958-63.

5. Lopez VJ, Galano GJ, Black CM, Gupta AT, James DE, Kelleher KM, et al. Profile of an American amateur rugby union sevens series. Am J Sports Med. 2012;40(1):179-84.

6. Taylor AE, Fuller CW, Molloy MG. Injury surveillance during the 2010 IRB Women's Rugby World Cup. Br J Sports Med. 2011;45(15):1243-5.

7. Ocwieja KE, Mihalik JP, Marshall SW, Schmidt JD, Trulock SC, Guskiewicz KM. The effect of play type and collision closing distance on head impact biomechanics. Ann Biomed Eng. 2012;40(1):90-6.

8. Bleakley C, Tully M, O'Connor S. Epidemiology of adolescent rugby injuries: a systematic review. J Athl Train. 2011;46(5):555-65.

9. Hollis SJ, Stevenson MR, McIntosh AS, Shores EA, Collins MW, Taylor $\mathrm{CB}$. Incidence, risk, and protective factors of mild traumatic brain injury in a cohort of Australian nonprofessional male rugby players. Am J Sports Med. 2009;37(12):2328-33.
10. Koh JO, Cassidy JD, Watkinson EJ. Incidence of concussion in contact sports: a systematic review of the evidence. Brain Inj. 2003;17(10):901-17.

11. Dick RW. Is there a gender difference in concussion incidence and outcomes? Br J Sports Med. 2009;43 Suppl 1:146-50.

12. Covassin T, Elbin RJ, Harris W, Parker T, Kontos A. The role of age and sex in symptoms, neurocognitive performance, and postural stability in athletes after concussion. Am J Sports Med. 2012;40(6):1303-12.

13. McLellan TL, McKinlay A. Does the way concussion is portrayed affect public awareness of appropriate concussion management: the case of rugby league. BrJ Sports Med. 2011;45(12):993-6.

14. Daneshvar DH, Nowinski CJ, McKee AC, Cantu RC. The epidemiology of sport-related concussion. Clin Sports Med. 2011;30(1):1-17.

15. Cantu RC, Gean AD. Second-impact syndrome and a small subdural hematoma: an uncommon catastrophic result of repetitive head injury with a characteristic imaging appearance. J Neurotrauma. 2010;27(9):1557-64.

16. De Beaumont L, Mongeon D, Tremblay S, Messier J, Prince F, Leclerc $S$, et al. Persistent motor system abnormalities in formerly concussed athletes. JAthl Train. 2011;46(3):234-40.

17. Gavett BE, Stern RA, McKee AC. Chronic traumatic encephalopathy: a potential late effect of sport-related concussive and subconcussive head trauma. Clin Sports Med. 2011;30(1):179-88.

18. McKee AC, Cantu RC, Nowinski CJ, Hedley-Whyte ET, Gavett BE, Budson AE, et al. Chronic traumatic encephalopathy in athletes: progressive tauopathy after repetitive head injury. J Neuropathol Exp Neurol. 2009;68(7):709-35.

19. Saunders RL, Harbaugh RE. The second impact in catastrophic contact-sports head trauma. JAMA. 1984;252(4):538-9.

20. Shuttleworth-Edwards AB, Smith I, Radloff SE. Neurocognitive vulnerability amongst university rugby players versus noncontact sport controls. J Clin Exp Neuropsychol. 2008;30(8):870-84.

21. Khurana VG, Kaye AH. An overview of concussion in sport. J Clin Neurosci. 2012;19(1):1-11.

22. Zafonte R. Diagnosis and management of sports-related concussion: a 15-year-old athlete with a concussion.JAMA. 2011;306(1):7986.

23. Cantu RC. Posttraumatic Retrograde and Anterograde Amnesia: Pathophysiology and Implications in Grading and Safe Return to Play. JAthl Train. 2001;36(3):244-8.

24. Maddocks DL, Dicker GD, Saling MM. The assessment of orientation following concussion in athletes. Clin J Sport Med. 1995;5(1):32-5.

25. Ropper AH, Gorson KC. Clinical practice. Concussion. N Engl J Med. 2007;356(2):166-72.

26. Makdissi M, Collie A, Maruff P, Darby DG, Bush A, McCrory P, et al. Computerised cognitive assessment of concussed Australian Rules footballers. BrJ Sports Med. 2001;35(5):354-60.

27. Makdissi M, McCrory P, Ugoni A, Darby D, Brukner P. A prospective study of postconcussive outcomes after return to play in Australian football. Am J Sports Med. 2009;37(5):877-83.

28. Asplund CA, McKeag DB, Olsen CH. Sport-related concussion: factors associated with prolonged return to play. Clin J Sport Med. 2004;14(6):339-43.

29. Haseler CM, Carmont MR, England M. The epidemiology of injuries in English youth community rugby union. Br J Sports Med. 2010;44(15):1093-9.

30. Frommer LJ, Gurka KK, Cross KM, Ingersoll CD, Comstock RD, Saliba SA. Sex differences in concussion symptoms of high school athletes. JAthl Train. 2011;46(1):76-84.

31. Alla S, Sullivan SJ, Hale L, McCrory P. Self-report scales/checklists for the measurement of concussion symptoms: a systematic review. BrJ Sports Med. 2009;43 Suppl 1:i3-12.

32. Elbin RJ, Schatz P, Covassin T. One-year test-retest reliability of the online version of ImPACT in high school athletes. Am J Sports Med. 2011;39(11):2319-24.

33. Makdissi M, Darby D, Maruff P, Ugoni A, Brukner P, McCrory PR. Natural history of concussion in sport: markers of severity and implications for management. Am J Sports Med. 2010;38(3):46471.

34. Echemendia RJ, Herring S, Bailes J. Who should conduct and interpret the neuropsychological assessment in sports-related 
concussion? BrJ Sports Med. 2009;43 Suppl 1:i32-5.

35. Matheson GO, Shultz R, Bido J, Mitten MJ, Meeuwisse WH, Shrier I. Return-to-play decisions: are they the team physician's responsibility? Clin J Sport Med. 2011;21(1):25-30.

36. Sye G, Sullivan SJ, McCrory P. High school rugby players' understanding of concussion and return to play guidelines. BrJ Sports Med. 2006;40(12):1003-5.

37. Meehan WP, 3rd, d'Hemecourt P, Collins CL, Comstock RD. Assessment and management of sport-related concussions in United States high schools. Am J Sports Med. 2011;39(11):2304-10.

38. Hollis SJ, Stevenson MR, McIntosh AS, Shores EA, Finch CF. Com- pliance with return-to-play regulations following concussion in Australian schoolboy and community rugby union players. $\mathrm{Br} J$ Sports Med. 2012;46(10):735-40.

39. Shenouda C, Hendrickson P, Davenport K, Barber J, Bell KR. The effects of concussion legislation one year later--what have we learned: a descriptive pilot survey of youth soccer player associates. PM R. 2012;4(6):427-35.

40. Decq P, Chermann JF, Loiseau H, Pariente J, Touchon J, Mias L, et al. Rugby professionnel et traumatismes crâniens (commotions cérébrales): recommandations pour leur prise en charge en France. Journal de Traumatologie du Sport. 2011;28(4):227-42. 